\title{
MELHORA DA SAÚDE MENTAL ATRAVÉS DO DESENVOLVIMENTO FÍSICO NA FASE GERIÁTRICA EM TEMPOS DE COVID-r9
}

\author{
Gabrieli Riato de Souza ${ }^{1}$ \\ Higor Almeida ${ }^{2}$ \\ Igor Henrique Batista ${ }^{3}$ \\ Julianna Candido Leite ${ }^{4}$ \\ Katia Cristina Gomes ${ }^{5}$ \\ Lucimara Antonelli ${ }^{6}$ \\ Matheus Mendes Pascoal ${ }^{7}$
}

RESUMO: INTRODUÇÃO: O envelhecimento é uma mudança, que é compreendida como um processo natural na vida dos seres humanos e de acordo com a OMS, portanto este momento crítico em que o mundo está passando que é a pandemia causada pelo novo coronavírus COVID-i9 (Sars-Cov-2), afetou pessoas de vários países com diferentes faixas etárias, a população idosa foi a que apresentou uma maior taxa de complicações e mortalidade após ser contaminado pela COVID- 19. Portanto foram adotadas medidas de prevenção, visando principalmente o isolamento social, que também teve suas desvantagens afetando diretamente os idosos. $\mathrm{O}$ isolamento não afetou somente a saúde física do idoso, mas também sua saúde mental esocial, o estudo tem como proposta avaliar o estado e impactos da pandemia na saúde do idoso, levar orientações e medidas de prevenção que afetam diretamente ou indiretamente a saúde do idoso e evidenciar o que mais afeta essa população, trazer medidas que possa prevenir e melhorar sua qualidade de vida dos idosos neste momento de isolamento social. Utilizando-se meios para ofertar essas orientações e levar suporte no momento atual da pandemia, empregando informações por meios de tecnologias de comunicação, como os grupos de WhatsApp através do celular e das rádio. JUSTIFICATIVA: principal motivação para sustentar o presente projeto de pesquisa, consiste na importância que o tema possui para o cenário atual de isolamento social dos idosos na pandemia COVID-19,desta forma tornando o tema pertinente para o momento.A estimativa de crescimento de idosos com sintomas depressivos pelo isolamento social aumenta a urgência na busca de métodos que proporcionem sentimentos de felicidade, prazer e afeto positivo, essenciais para o idoso.Levando em conta a atual fragilidade no cenário da saúde, a pesquisa enfatiza a importância do tema para a área de geriatria.Em tempos turbulentos em termos de saúde como os atuais, inserir novos hábitos de saúde pode funcionar como um catalisador para mudanças no estilo de vida destes idosos. Garantindo assim, uma sociedade com um envelhecimento mais saudável tanto no estado físico, quanto cognitivo e emocional. OBJETIVO: Promover um plano de intervenção para o bem estar social e saúde mental da pessoa idosa através da atenção básica. METODOLOGIA: $O$ artigo trata-se de uma revisão integrativa realizada com base em pesquisas de artigos científicos, encontrados nas plataformas da BVS e SCIELO, utilizando de descritores booleanos AND para otimizar a pesquisa.

PALAVRAS-CHAVE: Saúde. Idoso. Isolamento Social. Pandemia e COVID-I9.

\footnotetext{
${ }^{I}$ Acadêmica de Enfermagem do Centro Universitário Integrado (CEI).

${ }^{2}$ Acadêmico de Enfermagem do Centro Universitário Integrado (CEI).

${ }^{3}$ Acadêmico de Enfermagem do Centro Universitário Integrado (CEI).

${ }^{4}$ Acadêmica de Enfermagem do Centro Universitário Integrado (CEI).

${ }^{5}$ Acadêmica de Enfermagem do Centro Universitário Integrado (CEI).

${ }^{6}$ Acadêmico de Enfermagem do Centro Universitário Integrado (CEI).

${ }^{7}$ Graduando em Enfermagem-Centro Universitário Integrado, editor Júnior da Sábios, Membro da LAESM

e LADCIST.E-mail: matheus_mendesıs@hotmail.com
} 
ABSTRACT : INTRODUCTION: Aging is a change, which is understood as a natural process in the lives of human beings and according to the WHO, therefore this critical moment in which the world is going through is the pandemic caused by the new coronavirus COVID-i9 (Sars-Cov -2), affected people from various countries with different age groups, the elderly population was the one with the highest rate of complications and mortality after being contaminated by COVID- 19. Therefore, preventive measures were adopted, aiming mainly at social isolation, which it also had its disadvantages directly affecting the elderly. Isolation not only affected the physical health of the elderly, but also their social and social health, the study aims to assess the state and impacts of the pandemic on the health of the elderly, to take guidance and preventive measures that directly or indirectly affect the health of the elderly and highlight what most affects this population, bring measures that can prevent and improve the quality of life of the elderly in this moment of social isolation. Using means to offer these guidelines and take support in the current moment of the pandemic, using information by means of communication technologies, such as WhatsApp groups via cell phones and radio. BACKGROUND: main motivation to support the present research project, is the importance that the theme has for the current scenario of social isolation of the elderly in the pandemic COVID-I9, thus making the topic relevant for the moment. with depressive symptoms due to social isolation increases the urgency in the search for methods that provide feelings of happiness, pleasure and positive affection, essential for the elderly. Taking into account the current fragility in the health scenario, the research emphasizes the importance of the theme for the area In turbulent times in terms of health such as the present, inserting new health habits can act as a catalyst for changes in the lifestyle of these elderly people. Thus guaranteeing a society with a healthier aging both in the physical, as well as cognitive and emotional. OBJECTIVE: To promote an intervention plan for the social well-being and mental health of the elderly through primary care. METHODOLOGY: The article is an integrative review carried out based on research of scientific articles, found on the platforms of the VHL and SCIELO, using Boolean descriptors AND to optimize the search.

Keywords: Health. Elderly. social isolation. pandemic and COVID-ig.

\section{INTRODUÇÃO}

O envelhecimento é uma mudança, que é compreendida como um processo natural na vida dos seres humanos e de acordo com a OMS, a população mundial está envelhecendo rapidamente. Com o decorrer do tempo, com melhores condições na qualidade de vida da população, ouve um aumento da expectativa de vida da população, resultando em uma grande acentuado aumento no número de idosos. Em virtude disso, a população idosa vem crescendo gradativamente e isso gera impactos socioeconômicos e a saúde pública em toda a sociedade. Ademais, com a pandemia COVID 19, ouve grande aumento na demanda nos hospitais, causando um sobrecarga na saúde pública, prejudicando assim ainda mais esse setor. Contudo, nesse tempo de isolamento social causado pela pandemia, os idosos são destaques na pandemia COVID 19,merecendo uma atenção especial, devido ao potencial risco dessa população.

$\mathrm{O}$ risco de morrer de COVID ig é maior com pessoas idosas, especialmente aqueles 
com doenças crônicas, contudo o Brasil e o mundo adotaram medidas de isolamento e distanciamento social e comportamentos preventivos individual e coletivo de higienização constante, como lavagem das mãos, uso de álcool em gel, distanciamento entre as pessoas e o uso de máscaras.

A vivencia durante a pandemia COVID 19, tem destacado a importância com o cuidado e a atenção com os idosos, porque com o envelhecimento, vai começando aparecer o comprometimento mental e físico evidenciado principalmente por alterações mentais, ansiedade, insônia, irritabilidade, pressão alta, diabetes e etc. Entre os diversos problemas de saúde da pessoa idosa, damos o foco, nos problemas relacionados a saúde mental do idoso, porque, com a idade mais avançada acontece muitas mudanças em sua vida principalmente a condição física e para lidar com esse processo naturalmente, primeiro é preciso entender a importância da saúde mental do idoso.

Assim, muitas doenças crônicas podem ser evitadas e a qualidade de vida do idoso melhorada, e é de suma importância prestar atenção nas atitudes e comportamento dos idosos para que possam servir de informações relevantes para o cuidado com a saúde mental do idoso. Nesse sentido, são importantes o acolhimento, prevenção e a promoção da saúde, com vista, assegurar mais qualidade de vida aos idosos e bem estarda população como um todo.

Considerando o momento epidemiológico, conforme Forattini (1992), qualidade de vida é um conceito difícil de ser definido, sendo, por isso, muito mais difícil de ser medido. Levando em consideração tal realidade que a população tem passado, estima-se que a ansiedade, depressão estão entre os principais problemas de saúde na população idosa durante a pandemia e entre os principais agravos que implicam na diminuição da autonomia, independência e funcionalidade nos idosos, assim como na realidade socio vivencial e qualidade de vida (Moraes, 2012; WHO, 2012).

A depressão é um sofrimento que causa a alterações negativas nas atividades do diaa-dia que na maioria dos problemas a ansiedade e depressão podem ser resolvidos com o acolhimento e diagnóstico precoce, mas na população idosa normalmente passa despercebida e por isso, pouco tratada na saúde primária. Entre outros fatores temos a perda dos entes queridos, a mudança forçada de domicílio, situações de desamparo, solidão e abandono. Além disso, as enfermidades físicas crônicas, terminais e incapacitantes como, por exemplo, Alzheimer e Parkinson, abuso de álcool e outras drogas também estão relacionados ao comprometimento de saúde. Sob essa ótica, a comunicação com a população idosa é fundamental e necessita de articulações multiprofissionais e interdisciplinares sobretudo, no campo da Atenção primariaque é a porta de entrada ao sistema único de saúde (SUS).

Nesse sentido, com relação a questão de adaptação ao isolamento social vem gerando muito impacto na população em geral e principalmente a população idosa é necessário buscar medidas de prevenção e promoção a saúde do idoso.

Sendo assim, como se adaptar ao isolamento social e cuidar da saúde mental da pessoa idosa? Vale ressaltar, que devido á fácil transmissão pela corona vírus, a recomendação das autoridades no momento é manter o distanciamento social. Dessa forma, a saúde mental do idoso pode ser melhorada através da promoção de um envelhecimento ativo e saudável envolvendo a criação de âmbitos de vida como boa alimentação, exercícios físicos e bem estar-social que permitam que os idosos tenham uma vida saudável e autônoma durante e após essa pandemia. 
Então, para melhorar a saúde mental da população idosa é importante assegurar que os idosos tenham recursos necessários para atender às suas necessidades como o desenvolvimento de novas abordagens terapêuticas, psicológicas, boa alimentação, exercícios físicos e das tecnologias em geral.

A tecnologia tem se mostrado um grande aliado da saúde mental do idoso, na medida em que mantém ativa a atividade intelectual e cognitiva dos idosos as, redes socias, vídeos, notícias e mensagens que pode ser usado como ferramenta no tratamento e entretenimento da pessoa idosa que pode ser acessado dentro de casa.

Ademais, é importante desenvolver ações educativas relativas à saúde do idoso de acordo com o planejamento da equipe, realizar assistência domiciliar, realizar consulta de enfermagem e orientar ao idoso, aos familiares e ao cuidador sobre a correta utilização dos medicamentos. Envolver o idoso em atividades e programas de exercícios físicos que pode ser transmitido pelo celular, computador ou televisão, promovendo saúde e alegria ao idoso dentro de casa ou até mesmo no quintal e envolta da casa. Assim, contribuir para identificar as causas e ações possíveis de direcionamento ao risco de solidão, procurando ajudá-los na adaptação dessa situação.

Relacionado ao ambiente aonde vive o idoso é importante estabelecer uma rotina bem estabelecida para que os idosos tenham horário para comer, descansar e dormir todo dia no mesmo horário. Além de promover apoio em atividades de laser para o idoso é de suma importância escutar atentamente as preocupações dos idosos procurando intender as suas dúvidas e preocupações e principalmente ter o contato com amigos e familiares seja por ambiente virtual ou físico dependendo do número de contaminados em sua cidade e respeitando o distanciamento e o uso de máscaras de proteção. $O$ momento de distanciamento social é uma medida de precaução e proteção a saúde, porém, a autonomia e a independência dos idosos é fundamental para um envelhecimento saudável.

Dado o exposto, durante a período de pandemia COVID i9, se viu a importância e o destaque ao ensino geriátrico na formação profissional e a qualificação para o cuidado ao idoso. Portanto, para a enfermagem, surge a necessidade da formação dos profissionais de enfermagem mais qualificados no modelo da atenção psicossocial, buscando apoia dos gestores municipais e familiares para os desafios relacionados a assistência a pessoa idosa.

\section{I.I Problema}

Como se adaptar ao isolamento social e cuidar da saúde mental da pessoa idosa?

\section{I.2 Justificativa}

A principal motivação para sustentar o presente projeto de pesquisa, consiste na importância que o tema possui para o cenário atual de isolamento social dos idosos na pandemia COVID-ı; Desta forma tornando o tema pertinente para o momento.

A estimativa de crescimento de idosos com sintomas depressivos pelo isolamento social aumenta a urgência na busca de métodos que proporcionem sentimentos de felicidade, prazer e afeto positivo, essenciais para o idoso.

Levando em conta a atual fragilidade no cenário da saúde, a pesquisa enfatiza a importância do tema para a área de geriatria.

Em tempos turbulentos em termos de saúde como os atuais, inserir novos hábitos 
de saúde pode funcionar como um catalisador para mudanças no estilo de vida destes idosos. Garantindo assim, uma sociedade com um envelhecimento mais saudável tanto no estado físico, quanto cognitivo e emocional.

\subsection{Objetivos}

\subsection{Objetivo geral}

Promover um plano de intervenção para o bem estar social e saúde mental da pessoa idosa através da atenção básica.

\subsubsection{Objetivos específicos}

- Avaliar/identificar o estado de saúde mental e envolver os profissionais de saúde, a família e a comunidade destes pacientes.

- Orientar a importância da prevenção e medidas específicas de isolamento social para a saúde do idoso. de pandemia.

- Evidenciar os parâmetros que afetam a saúde do idoso no momento

\section{METODOLOGIA}

\begin{tabular}{|l|l|}
\hline $\begin{array}{l}\text { OBJETIV } \\
\text { O I }\end{array}$ & Evidênciar os parâmentros que afetam a saúde dos idosos no momento de pandemia \\
\hline O que? & $\begin{array}{l}\text { Incentivar os idosos na realização de atividades e exercícios físicos para melhora da } \\
\text { saúde cognitiva e funcional }\end{array}$ \\
\hline Onde? & Em meios domiciliares; \\
\hline Por que? & $\begin{array}{l}\text { Para identificar os problemas que afetam a saúde mental da pessoa idosa, procurando } \\
\text { ajuda-los na adaptação durante a pandemia }\end{array}$ \\
\hline Quando? & Tempo indeterminado \\
\hline Quem? & Equipe de saúde \\
\hline Como? & Orienta-los através de: redes sociais; rádio; cartilhas educativas; teleconsulta. \\
\hline Quanto? & Sem custo \\
\hline Avaliação & $\begin{array}{l}\text { Realizar a avaliação através da Sistematização de Enfermagem por meio de visitas } \\
\text { domiciliares (pacientes críticos) e Teleconsulta (pacientes de risco habitual) }\end{array}$ \\
\hline
\end{tabular}

OBJETIV Orientar sobre prevenção e medidas significativas perante ao isolamento social

O II

O que? Manter isolamento social e adotar medidas preventivas tais como: Lavar as mãos; usar alcool a 70\% sempre que possível e adotar uso de máscara.

Onde? $\quad$ Em meios domiciliares e em locais públicos

Por que? Para minimizar os riscos de contaminação e agravos diante de suas condições crônicas

Quando? Tempo indeterminado

Quem? Equipe de saúde

Como? Orienta-los através de: redes sociais; rádio; cartilhas educativas e por teleconsulta

Quanto? Sem custo 
Avaliação Através de um feed back dado pelo paciente ao enfermeiro (feito uma avaliação através da Sistematização de Enfermagem por meio de visitas domiciliares) (pacientes críticos) ou então utilização de redes sociais, tais como whatsapp ou teleconsulta (pacientes de risco habitual)

\begin{tabular}{|l|l|}
\hline $\begin{array}{l}\text { OBJETIV } \\
\text { O III }\end{array}$ & Avaliar e identidicar o estado de saúde mental dos idosos \\
\hline O que? & $\begin{array}{l}\text { Melhorar a saúde mental dos idosos através do desenvolvimento de novas abordagens } \\
\text { terapeuticas, psicológicas etc. }\end{array}$ \\
\hline Onde? & Domiciliar; e se caso houver necessidade em unidades de saúde. \\
\hline Por que? & $\begin{array}{l}\text { Para deteçãa precoce de problemas e intervenção para prevenção de degeneração e } \\
\text { melhorar a qualidade de vida. }\end{array}$ \\
\hline Quando? & Tempo indeterminado \\
\hline Quem? & Equipe de Saúde \\
\hline Como? & Por teleconsultas,via redes socias, cartilhas e se necessário por consulta presencial. \\
\hline Quanto? & Sem custo \\
\hline Avaliação & $\begin{array}{l}\text { Pode ser realizada através da escala de blessed; Escala de avaliação cognitiva nos } \\
\text { idosos, realizada de forma geral pelo enfermeiro(a) avaliando o grau de dependência e } \\
\text { de conciência do idoso }\end{array}$ \\
\hline
\end{tabular}

\section{EFERÊNCIAS}

BRAGA, Marisa Moreira. et al. Proposta de um protocolo de treino e seu efeito nas funções cognitivas em idosas depressivas. R. bras. Ci. e Mov 2019;27(3):25-33.

Brasil. Ministério da saúde. Secretaria de Atenção Primária à Saúde. Departamento de Ações Programáticas Estratégicas. Coordenação-Geral de Ciclos da Vida. Coordenação de Saúde das Mulheres. Nota Técnica no ro/2020-COSMU/CGCIVI/DAPES/SAPS/MS Recomendações para as Consultas Ambulatoriais de Saúde da Mulher durante a Pandemia da COVID-19. Abr., 2020.

FERREIRA, Joel Saraiva; DIETTRICH Sandra Helena Correia; PEDRO, Danielly Amado. Influência da prática de atividade física sobre a qualidade de vida de usuários do SUS. Saúde Debate. Rio de Janeiro, V. 39, N. Io6, p. 792-8or, jul-set 2015.

GARCIA, Bruno Nogueira; MOREIRA, Daiana de Jesus; OLIVEIRA, Pedro Renan Santos de. Saúde mental do idoso na atenção primária: Uma análise das percepções de profissionais de saúde. Revista Kairós - Gerontologia, 2o(4), São Paulo-SP, Brasil: FACHS/NEPE/PEPGG/PUC-SP. p.153-174, 2017.

HAMMERSCHMIDT, Karina Silveira de Almeida; SANTANA, Rosimere Ferreira. Saúde do idoso em tempos de pandemia Covid-r9. Cogitare enferm. [Internet]. 2020 [acesso em 19/10/2020; 25. Disponível em: http://dx.doi.org/ro.5380/ce.v25io.72849.

HERNANDEZ, José Augusto Evangelho, VOSER, Rogério da Cunha.Exercício regular e 
depressão em idosos. Rio de Janeiro: Revista: Estudos e Pesquisas em Psicologia, v.19, n. 3 2019.

OLIVEIRA, Daniel Vicentini de. et al. Investigação dos fatores psicológicos e emocionais de idosos frequentadores de clubes de dança de salão. Rev. Bras. Geriatr. Gerontol., Rio de Janeiro, 2017; 20(6): 802-810.

SILVA, Vanessa Regina da; SOUZA, Guilherme Rodrigues de; ALVES, Silvia Cristina Crepaldi. Benefícios do exercício físico sobre as alterações fisiológicas, aspéctos sociais, cognitivos e emocionais no envelhecimento. Revista CPAQV - Centro de Pesquisas Avançadas em Qualidade de Vida. Vol. 7. № 3, 2015. P.I2.

ZEN, Daniela, et al. Políticas de atenção a idosos na voz de gestores municipais de saúde. Porto Alegre: Rev. Gaúcha Enferm. vol.39 Epub 23-Jul-2018. 